\title{
Three-dimensional cell culture: the missing link in drug discovery
}

\section{Susan Breslin and Lorraine O'Driscoll}

School of Pharmacy and Pharmaceutical Sciences \& Trinity Biomedical Sciences Institute, Trinity College Dublin, Dublin 2, Ireland

Cells, grown as monolayers (2D models), are routinely used as initial model systems for evaluating the effectiveness and safety of libraries of molecules with potential as therapeutic drugs. While this initial screening precedes preclinical animal studies before advancing to human clinical trials, cultured cells frequently determine the initial, yet crucial, 'stop/go' decisions on the progressing of the development of a drug. Growing cells as three-dimensional (3D) models more analogous to their existence in vivo, for example, akin to a tumour, and possibly co-cultured with other cells and cellular components that naturally occur in their microenvironment may be more clinically relevant. Here, in the context of anticancer drug screening, we review 2D and 3D culture approaches, consider the strengths and relevance of each method.

\section{The need for more efficient and cost-effective preclinical screening of anti-cancer drugs}

The design and development of all new drugs, for the most part, follow a similar trend of progression. Typically a potentially druggable target is identified and, often with the aid of in silico modelling, lead compounds are designed, developed and optimised to act on this target. Preclinical testing is then performed with compound libraries to establish which members of the library exhibit efficacy towards the target in question. Preclinical testing involves both in vitro analyses in appropriate cell line models, as well as in vivo studies in relevant animal models. These are performed to determine toxicity, in addition to pharmacokinetic and pharmacological characteristics testing to investigate absorption, distribution, metabolism and excretion properties; fundamental in determining the basic safety and potential usefulness of the drug. Finally, clinical testing of suitable compounds, through various stages of clinical trials, is performed in humans; an essential step to evaluate the ultimate usefulness of the drug.

While there has been an increase in the number of potential anti-cancer agents being advanced for development over the past 10 years [1], the number of these products that progress successfully throughout clinical development is low, at approximately $10 \%$ [2]. Lack of clinical efficacy and/or unacceptable toxicity are two of the main causes of drug failures during development $[3,4]$. Because of the high costs (typically $\sim 1$ billion US dollars) in getting new drugs approved and the fact that many oncology drugs fail during clinical testing, especially during phase III - the most expensive phase of clinical development $[5,6]$, it is imperative that compounds that are potentially ineffective or have an unacceptable toxicity profile are dismissed as early in the evaluation process as possible. This would preferably be before clinical trials and, ideally, even before animal testing has begun. Failing early in the developmental stages enables the cost of failed molecules to remain relatively low, that is, the further into the development process a compound fails, understandably the more money a company has invested and so stands to lose [7]. It is, therefore, necessary to improve in vitro cell-based testing methods for a more informed prediction of drug candidate efficacy and safety, and thereby sieve out poorly functioning compounds while prioritising promising candidates [6].

The ability of an in vitro assay to produce reliable biomedically relevant information is essential in drug development; therefore, it is necessary that the cells used in this testing mimic the phenotype of cells within the target tissue $[7,8]$. 2D cell culture (i.e. monolayer culture) is conventionally used in in vitro drug candidate testing; however, limitations of $2 \mathrm{D}$ culture suggest that an alternative method should be considered. These limitations include the lack of cell-cell and cell-extracellular matrix (ECM) signalling that 
occurs in the 3D (three-dimensional, multi-cellular spheroids) in vivo environment where such signals are essential to cell differentiation, proliferation and a range of cellular functions $[9,10]$. For example, integrins, which are cell surface receptors, anchor cells to the ECM and are also involved in the cells' interpretation of biochemical cues from their local environment [11,12]. It is probable, therefore, that $3 \mathrm{D}$ cellular assays would be more analogous to - and so predictive of - in vivo events compared to more simplified $2 \mathrm{D}$ cultures in which essential signalling pathways may have been lost or, at least, compromised [13]. The use of 3D in vitro systems in drug research and development has, therefore, been suggested as a potential link to bridge the gap between monolayer cultures and animal model studies [6,14].

This article reviews both $2 \mathrm{D}$ and $3 \mathrm{D}$ cell culture. The 3D procedures discussed include methods that modify cell culture surfaces and thereby promote 3D culture formation by preventing cells from attaching to their surface; the hanging drop method which supports cellular growth in suspension; rotary systems that encourage cells to adhere to each other to form 3D spheroids; 3D scaffolds and matrices, which provide extracellular support and allow 3D cell growth; and microfluidic systems that support 3D cell culture. While the same methods are relevant regardless of the drug types being assessed, a comprehensive review of all applications of 2D and 3D cultures is not possible; so in the context of our own research here we focus on model systems for screening anticancer drugs; particularly in the context of breast cancer. Throughout this review we highlight the differences between each culture method. We propose the necessity to incorporate 3D cell methods into drug development for human therapy, based on their ability to mimic tissue-like structures more effectively than 2D cell culture. Advantages and disadvantages of various methods for 3D culture are subsequently summarised in Table 1 .

\section{A brief history of cell culture in 2D}

The process of cell culturing was developed, in 1907, by Harrison while investigating the origin of nerve fibres [15]. Specifically, explanted pre-differentiated neural tissue from frog embryos was placed in a drop of lymph hanging from a sterile cover-slip, kept sealed and in a moist chamber. This method allowed tissue growth and differentiation to be continually observed [16]; demonstrating a means by which cells of interest could be maintained outside the body of origin and observed over time.

Substantial improvements have been made on the $2 \mathrm{D}$ cell culture technique initially developed by Harrison. Containers used for culturing have been developed which enable cells to be fed with ease and that allow more space for cell growth. Additionally, the traditional use of blood plasma as the only source of nutrition for the growing cells changed to the use of synthetic medium. There are many advantages including the fact that batches of synthetic medium can be made reproducibly; do not contain antigens which can cause allergic reactions; and are relatively cheap to produce. Antibiotics and anti-fungal agents have been developed that are suitable for cell cultures and thus help to prevent bacteria and fungi from infecting cultures. While these additives are not a substitute for good cell culture practice, they can be useful for maintenance of infection-free cells, if contamination is envisaged to be a problem.

Cells are typically grown as a monolayer on a flat surface, most commonly in culture flasks or sometimes in Petri-dishes with medium as a source of nutrition and at body temperature $\left(37^{\circ} \mathrm{C}\right)$. Medium is often supplemented with bovine serum and L-glutamine to aid cell growth. When reaching confluency, cells are sub-cultured so as to avoid complications from senescence or nutrient-exhaustion from medium. To sub-culture, cells are cleaved from the bottom of their culture dish (with trypsin and/or EDTA) and a quantity

TABLE 1

\begin{tabular}{|c|c|c|}
\hline \multicolumn{3}{|c|}{ Proposed advantages and disadvantages of different 3D cell culture methods } \\
\hline Method type & Advantages & Disadvantages \\
\hline Forced-floating & $\begin{array}{l}\text { - Relatively simple } \\
\text { - Inexpensive } \\
\text { - Suitable for high-throughput testing } \\
\text { - Spheroids produced are easily accessible }\end{array}$ & $\begin{array}{l}\text { - Variability in cell size and shape if not as fixed } \\
\text { cell no./well } \\
\text { - DIY plate-coating is relatively labour intensive }\end{array}$ \\
\hline Hanging drop & $\begin{array}{l}\text { - Inexpensive if using standard 96-well plate } \\
\text { - Homogenous spheroids suitable for } \\
\text { high-throughput testing } \\
\text { - Spheroids produced are easily accessible }\end{array}$ & $\begin{array}{l}\text { - More expensive if using specialised plates } \\
\text { - Labour intensive if preparing plates in-house } \\
\text { - Small culture volume makes medium exchange, } \\
\text { without disturbing cells, difficult (proposed easier } \\
\text { handling with commercially available formats) }\end{array}$ \\
\hline $\begin{array}{l}\text { Agitation-based } \\
\text { approaches }\end{array}$ & $\begin{array}{l}\text { - Simple to culture cells } \\
\text { - Large-scale production relatively easily achievable } \\
\text { - Motion of culture assists nutrient transport } \\
\text { - Spheroids produced are easily accessible }\end{array}$ & $\begin{array}{l}\text { - Specialised equipment required } \\
\text { - No control over cell no./size of spheroid (can be } \\
\text { overcome by additional culture step; see 'Forced-floating methods') } \\
\text { - Time consuming for HTS due to extra step } \\
\text { required for homogenous spheroids } \\
\text { - Cells possibly exposed to shear force in spinner } \\
\text { flasks (may be problematic for sensitive cells) }\end{array}$ \\
\hline $\begin{array}{l}\text { Matrices and } \\
\text { scaffolds }\end{array}$ & $\begin{array}{l}\text { - Provide 3D support that mimics in vivo } \\
\text { - Some incorporate growth factors }\end{array}$ & $\begin{array}{l}\text { - Can be expensive for large-scale production } \\
\text { - Can have difficulty in retrieving cells following } \\
\text { 3D culture formation }\end{array}$ \\
\hline $\begin{array}{l}\text { Microfluidic cell } \\
\text { culture platforms }\end{array}$ & - Described as suitable for high-throughput testing & $\begin{array}{l}\text { - Specialised equipment required adding expense } \\
\text { - Further analysis of 3D cultures produced may } \\
\text { be difficult }\end{array}$ \\
\hline
\end{tabular}


of the cells is re-seeded into a flask for continued growth of the cell line.

While continued development of this technique over the past century has been of fundamental importance, developments in the form of 3D cultures have highlighted some of the shortcomings of 2D monolayers. The correlation of results from $2 \mathrm{D}$ cultures to real-life in vivo scenarios has been questioned. Differences in cell morphology, polarity, receptor expression, oncogene expression, interaction with the ECM (including the basement membrane) and overall cellular architecture have been noted between cells grown as 2D monolayers and what is observed in vivo. As a result, more attention is shifting to $3 \mathrm{D}$ culture methods as it has been suggested and, indeed, verified in many reports, that cells grown in $3 \mathrm{D}$ are more representative of what occurs naturally in vivo. The differences between cells grown in 2D and 3D will be discussed in detail below (see 'Supporting evidence for the importance of including 3D cultures').

\section{D cell culture: adding a new dimension to drug discovery}

To improve drug development using 3D culture, the functional unit of tissues must be considered rather than single cells. Mammary tissue comprises epithelial cells that have a distinguished cellular architecture. These cells have specialised cell-cell contacts, a polarised morphology and are attached to an underlying basement membrane. The maintenance of these features is essential for normal function of the tissue, including proliferation, differentiation, survival and secretion $[17,18]$. The functional unit in breast tissue, as described by Bissell et al. [9], includes the cells plus their ECM. Therefore, the use of 3D cell culture, which allows the growth of multi-cellular 3D cultures that retain essential features of typical breast tissue and can (depending on the method) incorporate elements of ECM, may be invaluable to the drug development process. While the perfect method for 3D cell structures may not yet have been determined, methods have been developed based on our knowledge of cellular/tissue architecture, in efforts to produce the most in vivo-like structures possible. The more commonly used 3D culture methods are detailed below, while some newer methods are also discussed (Fig. 1 and Table 1).

\section{Forced-floating methods}

A relatively simple method for generating 3D spheroids is to prevent their attachment to the vessel surface by modifying the surface, resulting in forced-floating of cells (Fig. 1a). This promotes cell-cell contacts which, in turn, promotes multi-cellular sphere formation [19]. Using this approach, Ivascu and Kubbies [20] developed a method for producing 3D spheroids from cancerous and non-cancerous cells which enables the rapid production of spheroids in round or conical bottomed 96-well plates. In brief, plates were coated with $0.5 \%$ poly-2-hydroxyethyl methacrylate (poly-HEMA) and dried for three days before the addition of cells. The poly-HEMA prevents cells from attaching to the surface of the wells. In their studies, a cell suspension was seeded and plates were subsequently centrifuged to encourage cells to co-localise and thus adhere to each other. A panel of eight breast cancer cell lines (MCF7, T-47D, MDA-MB-435, MCF7-ADR, MDA-MB-231, MDAMB-468, SKBR3, MDA-MB-361) were tested. While some cell lines (MCF7, T47D, MDA-MB-435, MCF7-ADR) formed spheroids with this method, others (MDA-MB-231, MDA-MB-468, SKBR3, MDAMB-361) produced cell aggregates only, that is, where cells accumulated loosely together, but did not form tightly packed spheroids. The addition of $2.5 \%$ liquid reconstituted basement membrane (rBM) to suspensions of cell lines that produced aggregates circumvented this problem. This addition enabled all cell lines tested to generate compact 3D spheroids within 24 hours of centrifugation.

The forced-floating method of spheroid generation has many benefits. It is simple and generally reproducible, as equal numbers of cells can be seeded in each well for generation of consistent spheroids. Accordingly, the sizes of spheroids are adjustable, that is, for larger spheroids simply seed larger quantities of cells. The spheroids generated are easily accessible for experimentation on single spheroids where relevant, making this method compatible with high-throughput drug testing [20]. As these 3D spheroids are typically generated in a 96-well plate, large numbers of morphologically homogenous spheroids are easily produced. This is ideal for high-throughput investigations into the efficacy versus toxicity of drugs, gene expression in spheroids, and numerous other cellular and biochemical assays.

On the basis of the same principle of simply preventing cell attachment to the culture vessel, 3D cultures can be formed by using $1.5 \%$ agarose in culture medium to form a thin coating over culture dish surface. The agar prevents cell adhesion to the surface, thus resulting in the establishment of $3 \mathrm{D}$ spheroids [21,22]. The forced-floating method using agar is simple and inexpensive, and is proposed to allow for long-term culture of cells (more than 20 days) [21]. In fact, this method shares may of the advantages and limitations of the p-HEMA method [22].

A drawback with the forced-floating method, using either agar or poly-HEMA, is the time and work necessary to coat the plates before cell seeding. This adds an extra step to each assay performed, that is, whether using agar-coated plates which requires autoclaving of agar before its application [21] or poly-HEMA which takes a few hours to dissolve in 95\% ethanol (granted, this latter step need not be performed each time as p-HEMA can be prepared, aliquoted and stored). Precoated low cell adhesion plates are commercially available and so remove the need for including a plate-coating step; however, it must be considered that the purchase of precoated plates increases overall costs compared with inhouse precoating. Examples of commercially available precoated plates include the PrimeSurface low adhesion culture plate from Sumitomo Bakelite [23] and Lipidure-coated plates (Table 2) [24].

\section{Hanging drop method}

The hanging drop method (Fig. 1b) of 3D spheroid production is an adaption of the method used by Harrison. This method, adapted by Kelm et al. [25], uses a small aliquot (typically $20 \mu \mathrm{l}$ ) of a single cell suspension which is pipetted into the wells of a 60well MicroWell MiniTray (Nunc). Similarly to forced-floating, the cell density of the seeding suspension (e.g. 50, 100, 500 cells/well, among others) can be altered as relevant, depending on the required size of spheroids. Following cell seeding, the tray is subsequently inverted and aliquots of cell suspension turn into hanging drops that are kept in place due to surface tension. Cells accumulate at the tip of the drop, at the liquid-air interface, and are allowed to proliferate. Moisture levels are maintained using 


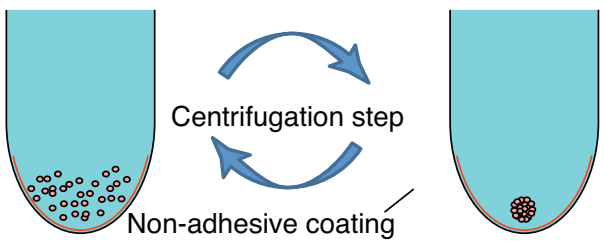

(a) Forced floating methods
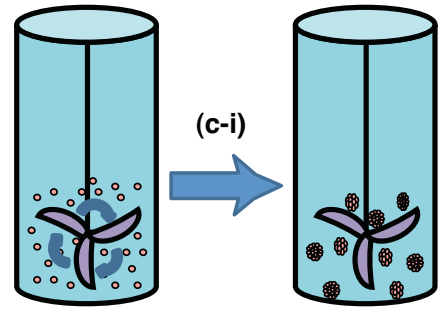

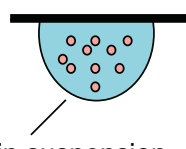

Cells in suspension

(b) Hanging drop methods

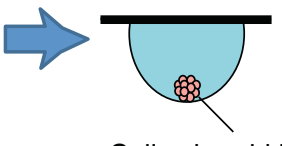

Cell spheroid in hanging drop

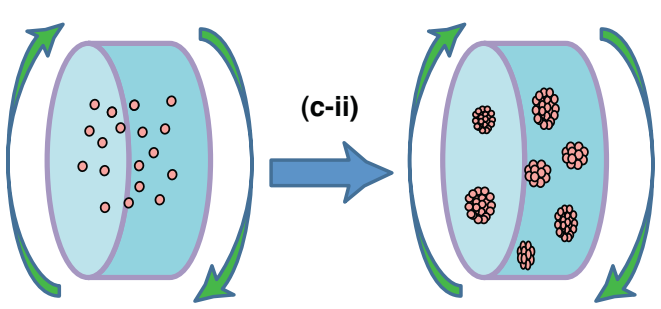

(c) Agitation based approaches

\section{- Growth}

factors

(d) Matrices and scaffolds- some available with growth factors

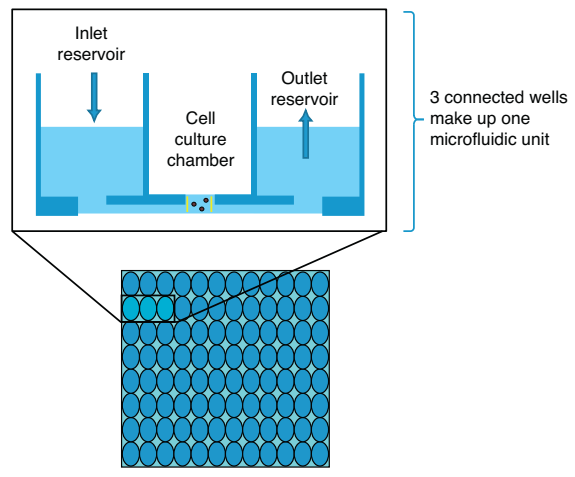

(e) Microfluidic system: MiCA plate

$\overline{\text { Drug Discovery Today }}$

FIGURE 1

Methods available for 3D multi-cellular spheroid formation. These methods include forced-floating of cells; hanging drop methods; agitation-based approaches; the use of matrices or scaffolds; and microfluidic systems.

bioassay dishes which act as moisture chambers and cells are incubated as per standard cell culture procedures.

The hanging drop method is relatively simple and has been reported to have a reproducibility of almost $100 \%$ for producing one 3D spheroid per drop, for numerous cell lines [25]. Spheroids produced are tightly packed spheroids, rather than loose cell aggregates, and they show low variability in sizes. Using HepG2 liver cancer cells and MCF-7 breast cancer cells as examples, Kelm et al. [25] reported that 3D spheroids produced were patho/physiologically relevant, as the resulting 3D structures are highly organised, produce their own ECM, and were thus described as 'tissue-like'. As this method is also based on cells natural tendency to adhere to each other as opposed to relying on matrices or scaffolds, this removes concerns regarding the effects that these products may have on the $3 \mathrm{D}$ structures formed. A potential drawback of the hanging drop, however, is the limitation of the volume of the liquid drop containing cells. This method can typically accommodate a volume of up to $50 \mu \mathrm{l}$ maximum (including the drug test medium), as the surface tension that keeps liquids attached to the culture surface does not support larger volumes [26]. Another limitation of the hanging drop method performed in this way is the difficulty in changing culture medium, due to difficulty in aspirating spent medium without disturbing the spheroid.

3D Biomatrix (http://3dbiomatrix.com/) have developed a 384well hanging drop plate that is claimed to support 3D cell culture on a larger scale [27]. This platform allows for large numbers of 3D spheroids to be produced using one plate. The system incorporates a bottom tray that includes a liquid reservoir to prevent evaporation/drying-out, a hanging drop plate that sits into the tray and 
TABLE 2

3D culture product information

\begin{tabular}{|c|c|c|}
\hline Method type & Product & Example relevant companies \\
\hline Forced-floating & $\begin{array}{l}\text { PrimeSurface low adhesion culture plate } \\
\text { Lipidure-coated plates } \\
\text { Happy Cell Media }\end{array}$ & $\begin{array}{l}\text { Sumitomo Bakelite - http://www.sumibe.co.jp/english } \\
\text { NOF Corporation - http://www.nofeurope.com } \\
\text { Happy Cell - http://www.happy-cell.com }\end{array}$ \\
\hline \multirow[t]{2}{*}{ Hanging drop } & Perfecta3D Hanging Drop Plates & 3D Biomatrix - http://3dbiomatrix.com \\
\hline & GravityPLUS platform & InSphero - http://www.insphero.com \\
\hline Agitation-based approaches & $\begin{array}{l}\text { Spinner Flasks } \\
\text { Spinner Flasks } \\
\text { Rotary Cell Culture Systems }\end{array}$ & $\begin{array}{l}\text { Wheaton - http://www.wheatonsci.com } \\
\text { Corning - http://www.corning.com } \\
\text { Synthecon - http://www.synthecon.com }\end{array}$ \\
\hline Matrices & $\begin{array}{l}\text { Matrigel } \\
\text { PathClear Grade Basement Membrane } \\
\text { ECM gel } \\
\text { ECL Cell Attachment Matrix } \\
\text { Geltrex }\end{array}$ & $\begin{array}{l}\text { BD Biosciences - http://www.bdbiosciences.com } \\
\text { Amsbio - http://www.amsbio.com } \\
\text { Sigma-Aldrich - http://www.sigmaaldrich.com } \\
\text { Millipore - http://www.millipore.com } \\
\text { Invitrogen - http://www.invitrogen.com }\end{array}$ \\
\hline Scaffolds & $\begin{array}{l}\text { Perfecta 3D Cell Culture Scaffolds } \\
\text { 3D Biotek 3D Insert Scaffolds } \\
\text { Cytodex } 3 \text { microcarrier beads } \\
\text { ProNectin F coated microcarrier beads } \\
\text { Global Eukaryotic Microcarriers } \\
\text { Alvetex Scaffolds }\end{array}$ & $\begin{array}{l}\text { 3D Biomatrix - http://3dbiomatrix.com } \\
\text { Sigma-Aldrich - http://www.sigmaaldrich.com } \\
\text { GE Heathcare -http://www.gehealthcare.com/eueu/ } \\
\text { Solohill - http://www.solohill.com/ } \\
\text { Global cell solutions - http://www.globalcellsolutions.com/ } \\
\text { Amsbio - http://www.amsbio.com }\end{array}$ \\
\hline Microfluidic cell culture platforms & $\begin{array}{l}\text { Microchannel } 5250 \text { Assay Plate } \\
\text { MiCA plate }\end{array}$ & $\begin{array}{l}\text { BellBrook Labs - http://www.bellbrooklabs.com } \\
\text { CellASIC - www.cellasic.com/3D }\end{array}$ \\
\hline
\end{tabular}

has access holes for cell suspension samples to be placed to form hanging drops, and a lid. While the issue of small sample volumes cannot be overcome by this new platform (volumes of $\sim 10-20 \mu \mathrm{l}$ are recommended), due to its design this format removes the difficulty experienced in traditional cell culture methods with regards to changing growth medium. Specifically, the plate is designed such that the top of the hanging drop is accessible and enables $5 \mu$ l of medium to be removed and $7 \mu l$ to be replaced at a time (compensating for medium evaporation) [27]. This hanging drop plate makes cell spheroid production and handling easier (than, e.g. using an inverted 60-well tray) and enables 3D cultures produced to be tested in a high-throughput manner.

InSphero (http://www.insphero.com/) has also developed a hanging drop plate for 3D cell culture. This is of similar design to the plate from 3D Biomatrix in that seeding and medium exchange can occur at an opening at the top of the drop. The InSphero plate system also includes a 'trap plate' which apparently allows for easy harvesting of cultures formed. The trap plate is placed under the cell culture plate, surplus medium is added to the hanging drops so that the drops get too heavy to remain attached to the culture surface and so drop into their respective wells of the trap plate. The trap plate has a non-adhesive coating to ensure cultures remain in suspension to allow further culture or assays to be performed.

\section{Agitation-based approaches}

Agitation-based approaches for the production of 3D spheroids can be loosely placed into two categorise as (i) spinner flask bioreactors $[19,28]$ and (ii) rotational culture systems [29]. The general principle behind these methods is that a cell suspension is placed into a container and the suspension is kept in motion, that is, either it is gently stirred or the container is rotated. The continuous motion of the suspended cells means that cells do not adhere to the container walls, but instead form cell-cell interactions.

Spinner flask bioreactors. Spinner flask bioreactors (typically known as 'spinners') (Fig. 1c-i) include a container to hold the cell suspension and a stirring element to ensure that the cell suspension is continuously mixed [28]. Spinners offer a simple method of 3D cell spheroid generation and, depending on the size of the bioreactor, can enable large yields of spheroids to be produced [19]. The design of the spinner/bioreactor facilitates a certain amount of control over culturing; for example, medium can frequently be changed to allow for long-term culture of 3D spheroids. Additionally, the motion of culture fluids that are fundamental to this method is thought to assist the transport of nutrients to, and waste products from, the spheroids [28]. A drawback of using a spinner flask is that the sheer force experienced by cells, due the motion of the stirring bar through the cell suspension, can adversely affect the cellular physiology [19]. Also, the medium requirements of spinner flasks are, understandably, much larger than those of stationary cultures such as plate-based approaches, that is, typically approximately $100-300 \mathrm{~mL}$ in spinner flasks (depending on the flask size) compared with approximately $15-20 \mathrm{~mL}$ for stationary approaches [30]. Furthermore, batches of 3D spheroids formed in spinner flasks are typically of a broad range of sizes and so would require subsequent manual selection to obtain a group of similarly sized 3D spheroids for analysis, if spheroids of the same/similar size are required for drug screening assays [19]. To overcome the issue of generating spheroids of non-uniform size, spheroids may initially be formed in agarose-coated wells of a 96-well plate as in 'Forced-floating methods' and subsequently transferred to spinner flasks for culturing [31]. This combined method of forced-floating followed by spinner flask allows 3D spheroids of equal size/cell number to be grown in an environment where nutrients and oxygen can be controlled 
relatively easily and prolonged culture times are possible [30]. For drug screening purposes 3D spheroids would, of course, have to be re-plated into suitable dishes (e.g. 96- or 384-well plates), overall resulting in a number of necessary consecutive steps before drug screening (i.e. 96-well plates for forced-floating to form uniform spheroids; then spinner flasks; then 96- or 384-well plates for drug screening), which adds to the labour involved. Commercially available spinner flasks include Wheaton Spinner Flasks and spinner flasks from Corning (Table 2).

Rotating cell culture bioreactors. Rotating cell culture bioreactors (Fig. 1c-ii) function by similar means as the spinner flask bioreactor but, instead of using a stirring bar/rod to keep cell suspensions moving, the culture container itself is rotated. The rotating wall vessel (RWV), developed by NASA in 1992, was designed to mimic microgravity and exert a low sheer force on cells in culture. It comprises a culture chamber(s) which screws onto a rotator that slowly rotates the chamber(s) about a horizontal axis. The constant motion prevents cells from adhering to the chamber walls and the speed of rotation can be adjusted to achieve optimal culture. For example, at the beginning of a culture when cells are still in single cell suspension, the culture chamber may be rotated at $15 \mathrm{rpm}$; however, once cells begin adhering to each other and forming larger 3D structures, the speed of rotations may be increased so as to keep heavier spheroids in suspension. As the culture chamber rotates, so does its contents; which results in low sheer force experienced by the contents of the chamber [29]. This low sheer force is one of the main advantages of using the NASA Rotary Cell Culture System. Other advantages and limitations of this bioreactor are similar to those of the spinner flask; it follows a relatively simple method, enables large-scale production of 3D cultures, and allows for long-term culture of 3D spheroids. Ease in changing culture medium is also claimed for this method [32]. The limitations of using the NASA bioreactor are that it requires specialised equipment and - unless a pre-96-well plate culture step is included, as described for spinner flasks - there is no way of controlling the number of cells contained within the spheroids produced, which may necessitate manual selection of approximately homogeneously sized spheroids [19]. Commercially available forms of this are available by Synthecon (http:// www.synthecon.com/) and retail as Rotary Cell Culture Systems (Table 2).

\section{Matrices}

As mentioned above, the make-up of mammary glands is complex and includes an organised branched network of ducts from epithelial cells [33]. Epithelial cells that make up mammary tissue develop in this highly organised manner by interacting with neighbouring cells and their ECM, including the basement membrane to which they are attached. Signals between these breast tissue components enable cells to arrange themselves in an appropriately organised way within the tissue. As ECM affects cellular organisation and cell function, 3D culture methods that incorporate ECM arguably help to better mimic in vivo situations, as they allow for cell-ECM interactions [e.g. Fig. 1d].

Matrigel (BD Biosciences) (Table 2) is an example of a commercially available ECM that can be used to support 3D culture. It is composed of Engelbreth-Holm-Swarm (EHS) mouse tumour cellderived basement membrane proteins which include collagen IV, laminin, perlecan, entactin, matrix metalloproteinase-2 and growth factors [34]. Accordingly, the use of Matrigel, which incorporates extracts of the basement membrane that are essential for cell differentiation, enables the generation of 3D breast cancer cell spheroids that may develop in a natural way, as they have required components for cellular signalling in their organised structure. Cells grown on, or in, ECM interact with each other in $3 \mathrm{D}$ and, as they continue to grow, they develop into structures similar to those found in the tissue from which the cells originated [34]. For example, MCF7 cells growing in Matrigel form a stromal support structure [28]. Examples of other commercially available forms of ECM include PathClear Grade Basement Membrane Extract (Amsbio); ECM gel (Sigma-Aldrich); ECL Cell Attachment Matrix (Millipore); Geltrex (Invitrogen) (Table 2).

There are two commonly used methods for spheroid generation in ECM. In the first, cells are embedded and grown within the gel; in the second, the cells are grown on top of the gel [35]. The use of ECM to culture 3D spheroids while arguably more cumbersome/ laborious than some other methods (e.g. simply seeding cell onto p-HEMA-coated plates followed by centrifugation) is still a relatively easy and, therefore, a popular method; particularly as sterile ECM is commercially available. Additionally, forms of ECM with variations in the standard components are available. For example, ECM variations that are depleted of specific growth factors, those with higher levels of collagen IV, or supplemented with cartilage matrix molecules, can be obtained; which allows for ECM types to be chosen according to experimental requirements [34]. A potential drawback of using this approach is that ECM is a biological substance and, therefore, its exact composition varies between batches. Also, the costs associated with purchasing the matrix must be taken into account when considering large-scale production of 3D spheroids for high-throughput drug testing [36]. Furthermore, spheroid sizes tend not to be uniform, which may be undesirable for reproducible high-throughput drug testing. Perhaps the biggest issue with using ECM for this purpose is that cells tend to be unevenly distributed throughout the product. This can result in spheroids overlapping with each other while in culture, which is undesirable when single spheroid analysis is required [36].

A modified protocol for growing cells in ECM is proposed to rectify this problem. Specifically this uses an array-based system applying soft lithography to produce a micropattern of squares or circles in the ECM. These individual microstructures are produced as part of a main platform and form buried wells or free-standing structures of ECM in these patterns upon - or within - which cells can be seeded and cultured as single spheroids. This compares favourably to the unorganised and overlapping 3D spheroids that can occur with typical 'embedded' or 'on top' growth of cells [36].

\section{Scaffolds}

Prefabricated scaffolds (Fig. 1d) can alternatively be used to aid 3D culture. Cells seeded into such scaffolds can migrate between fibres of the scaffolds and attach to them. The interstitial space between fibres is filled by cells as they grow and divide, forming 3D cellular structures [37]. Collagen, laminin, alginate [38] and other biodegradable materials are commonly used for this purpose [39]. These products can be processed to form hydrogels for use as 3D cell culture scaffolds. Gels that incorporate ECM molecules can 
apparently communicate in vivo-like cues to cells to enable in vivolike cell behaviour [40]. Hydrogels used in this manner are often porous. This allows oxygen, nutrients and drugs to reach the proliferating cells and also facilitates removal of waste molecules; thus providing appropriate cell culture conditions [41]. The size of pores in a hydrogel can be manipulated as necessary during production [42], enabling hydrogels to be engineered to suit experimental requirements, for example, for hypoxia or drugbased studies. An example of a hydrogel suitable for 3D culture is HuBiogel from Vivo Biosciences (http://www.vivobiotech.com/ ).

Hydrogels can also form the base for cell-compatible microcarriers, which are tiny spheres (generally $<500 \mu \mathrm{m}$ ) that have surface areas of up to $500 \mathrm{~cm}^{2} / \mathrm{g}$ that enables a large number of cells to be cultured at any one time using small volumes of medium [40]. Microcarriers can be coated with cell adhesion promoting substrates (e.g. gelatin, collagen) and are generally compatible for use in typical bioreactors for cell culture. Examples include Cytodex 3 microcarrier beads from GE Heathcare (http://www3.gehealthcare.com/en/Global_Gateway) and ProNectin F coated microcarrier beads from Solohill (http://www.solohill.com/) (Table 2). Global Cell Solutions (http://www.globalcellsolutions.com/) also supply microcarriers suitable for 3D culture. These Global Eukaryotic Microcarriers (GEMs) have an alginate core that also encorporates magnetic paticles and is covered in a layer of gelatin. They are designed for use in the BioLevitator benchtop incubator. These GEMs have the advantages of a hydrogel from the alginate core and have the ability to culture large cell numbers due to their large surface area. They also have the additional benefit of the magnetic elements in the core which allows for control over the cultures during media exchange or culture harvesting. The downside to these carriers is the requirement of specialised equipment.

\section{Microfluidic cell culture platforms}

Microfluidic platforms (Fig. 1e) may also be useful for drug screening (e.g. [43]); however, many of these microfluidic channel-based systems only support 2D cultures that, arguably, do not represent the in vivo environment [44]. Toh et al. [39] described a microfluidic system that supports 3D culture. This system comprises a main microfluidic channel within which is an array of micropillars through which a cell suspension is passed. The micropillar array is designed to immobilise passing cells within the pillars and supports their growth, thus enabling cell-cell interactions. A collagen matrix is then passed through the system and forms a thin layer over the cells, conforming to their shapes and allowing for cellmatrix interactions. This system is proposed to have potential for high-throughput drug testing and to be compatible with high content analysis, due to its ability to be imaged in multi-dimensions ( $x, y$ and $z$ ). Additionally, this system is claimed to enable qualitative and quantitative analysis, while minimising reagent volume [45]. Limitations of microfluidics platforms can include the lack of opportunity to retrieve and extensively characterise the cell spheroids formed. For other examples of microfluidic platforms that support 3D cell culture; see [46,47] and review [48].

The MiCA plate [by CellASIC (http://www.cellasic.com/)] and the iuvo Microconduit Array Platform [by BellBrook Labs (http:// www.bellbrooklabs.com/)] are examples of such commercially available products (Table 2). The MiCA plate comprises a 96-well plate format that consists of an array of 32 independent functional units. Each unit is composed of an inlet well, a culture chamber, and an outlet well - all of which are connected. Fluid flow through the microfluidic units is pump-free and is facilitated by gravity and surface tension. Cells are seeded onto the culture chamber, $300 \mu \mathrm{l}$ of medium is placed into the inlet well and $30 \mu \mathrm{l}$ into the outlet well. Gravity drives flow from the fuller inlet well through the culture chamber to the less full outlet well to balance out differences in pressure between the wells, thereby enabling pump-free flow through the unit. In this manner, continuous perfusion of the culture can be maintained by re-filling the inlet and emptying the outlet [49]. Cells can be resuspended in ECM before seeding to facilitate 3D culture. This is also feasible with the MiCA plate. This platform enables long-term perfusion experiments in $3 \mathrm{D}$, ease of access to cells through the open top culture chamber, and it requires small fluid volumes.

The iuvo Microconduit Array Platform uses passive pumping of fluid between two inlets (one large and one small) with a connecting channel. No specialised equipment is needed to perform assays using this technology, cells are easily viewed under a microscope and fluid samples required for use are small. A potential drawback for this method is that performing further analysis (e.g. immunoblot) of 3D samples produced following use of microfluidic plates could be difficult, due to the small sample volumes produced per plate.

\section{Supporting evidence for the importance of including 3D cultures}

Apart from the differences in the spatial arrangement of cells when grown in $2 \mathrm{D}$ or $3 \mathrm{D}$, there are a number of biological differences that contribute to how the cells are exposed and thus react to therapeutic agents. The substantial effects that the microenvironment of a cell can have on its behaviour are well documented $[9,10]$. For example, in a study by Weaver et al. [10], non-malignant HMT-3522 breast cells and malignant HMT-3522 type cells were grown in 3D using Matrigel. The normal cells formed organised, polarised acini, similar to those found in healthy breast tissue. The cancerous cells, however, formed disorganised, loose aggregates. Both normal and cancerous cell types were treated with antibodies against $\beta 1$-integrin, the surface receptor of which was found to be overexpressed on the cancerous cells. Treatment of the normal cells with these antibodies resulted in apoptosis, but treatment of cancerous cells resulted in an apparent reversal of phenotype back to the normal cell type, where cells appeared to lose their abnormal shape and growth patterns and become indistinguishable from normal cells. A similar result was not observed when the same cells were grown in 2D; indicating that extracellular cues can affect cellular phenotype and, in this case, cellular phenotype was proposed to be dominant over genotype [10].

Another example of an alteration of cellular architecture between $2 \mathrm{D}$ and 3D cells was observed in the growth of SKBR-3 cells that overexpress HER2, an oncogene found to be overexpressed in approximately $25 \%$ of breast tumours [50]. Cells grown as 3D spheroids using p-HEMA-coated plates had HER2 homodimers form; while in 2D cultures, HER2 formed heterodimers with HER3 [51]. Furthermore, in 3D versus 2D culture, epidermal growth factor receptor (EGFR) levels were slightly reduced; phosphorylation of HER2, HER3 and EGFR was enhanced; and 
activated Akt was downregulated. Finally, when a fixed concentration of trastuzumab (a monoclonal antibody that targets HER2) was added to the cells, 2D cultures underwent approximately $16 \%$ reduction in proliferation, whereas proliferation in 3D spheroids was reduced by a considerable $48 \%$. This indicates that the architecture of $3 \mathrm{D}$ spheroids differs from that in $2 \mathrm{D}$ and that the targeted anti-cancer drug, trastuzumab, has a dramatically different effect on cells grown in monolayer as compared to those in 3D, due to differences in the cell surface molecules and thus intracellular signalling events. Pickl and Ries [45] concluded the 3D cultures to be more representative of the HER2 signalling pathway in tumours in vivo.

This difference with sensitivity to drug exposure between cells grown in $2 \mathrm{D}$ and $3 \mathrm{D}$ has also been shown with classical chemotherapy drugs. Using the hanging drop method to generate 3D spheroids, Tung et al. [24] presented data indicating that A431.H9 cells grown in 2D and 3D show differences in viability when treated with the same concentrations of 5-fluorouracil (5FU) and tirapazamine (TPZ). In the case of 5-FU, 2D cultures were reduced to approximately $5 \%$ viability following a 96-hour treatment (5-FU; $10 \mu \mathrm{M})$, whereas 3D cells treated with the same concentration and duration remained at $75 \%$ viability; indicating that these $3 \mathrm{D}$ spheroids were more resistant to the antiproliferative effects of 5-FU. Conversely, cells treated for 96 hours with TPZ $(10 \mu \mathrm{M})$ in 2D exhibit cell viability of $72 \%$, whereas corresponding $3 \mathrm{D}$ cultures had a viability of just $40 \%$. In this case, the $2 \mathrm{D}$ cells were more resistant to the effects of TPZ. This was reasoned to have occurred due to the mechanism of TPZ which, as a hypoxiaactivated cytotoxin, was activated moreso in the $3 \mathrm{D}$ scenario as the core of the spheroids are hypoxic due to the limited ability of oxygen to diffuse to the centre of the spheroid. Consequently, TPZ was further activated in 3D cells due to the higher levels of hypoxic cells than in $2 \mathrm{D}$, resulting in $3 \mathrm{D}$ cells being more sensitive to the drug. These data show that the manner in which cells are cultured (i.e. 2D or 3D) can substantially alter the effect of a drug on the cells. Also, this research highlights the fact that cells in 3D do not necessarily exhibit a higher drug resistance, but that it is a combination of the specific drug and the cellular environment that influence the cells' reaction [27]. The hypoxic core described by Tung et al. [27] mimics the hypoxic core found in solid tumours that occurs due to poor oxygen diffusion into cells at the centre of a cancerous mass, as oxygen cannot diffuse past an approximate $200 \mu \mathrm{m}$ limit [52]. Up to $60 \%$ of solid tumours have hypoxic regions within their structure [53]. This further supports the 3D spheroid tumour models as relevant for investigating effects of drugs intended for use on solid tumours, as hypoxia has many effects in tumour biology, some of which can affect drug responses. For example, hypoxia can enhance receptor tyrosine kinase-mediated signalling, it can cause pro-survival alterations in expression of genes that suppress apoptosis, and it can affect DNA repair pathways [52].

The hypothesis that differences in culture environment can affect how cells will respond to drug treatment is supported by findings that cells grown as spheroids exhibiting polarised 3D architecture and show difference in resistance to apoptosis and chemotherapeutic drugs (in addition to those mentioned above) when compared to $2 \mathrm{D}$ controls [54]. For example Li et al. [47] showed that MCF10A cell variants in 3D exhibit a higher resistance to doxorubicin compared to 2D controls $[55,56]$; SA87, NCI-H460 and $\mathrm{H} 460 \mathrm{M}$ cells grown in $3 \mathrm{D}$ have higher resistance to 5-FU and doxorubicin than those in 2D [48]; and MCF-7 cells grown in 3D and then treated with tamoxifen are less susceptible to the cytotoxic effects of the drug than cells grown in the same tamoxifen concentrations, but in 2D [49].

In vivo, tumours can develop drug gradients, due to poor drug uptake by a tumour or poor distribution of drugs within a tumour. This phenomenon can reduce therapeutic effects of a drug at the target site $[57,58]$. The rate at which drugs diffuse into a cancerous mass effects the efficiency of treatment with that drug; therefore, it is an important pharmacokinetic feature that should be replicated in cell-based in vitro drug development assays. Thurber and Wittrup [58] discuss the distribution of drugs in tumours compared to 3D spheroids. While the variabilities noted as contributing to drug penetration of a tumour in vivo were found to be a function of concentration and exposure time, variabilities that effect drug penetration of multi-cellular spheroid included drug dose, antigen density (for antibody-based therapy), incubation time and spheroid size. Spheroids used for this study were generated using the hanging drop method and were found to closely replicate drug diffusion observed in the in vivo scenario. This indicated that 3D spheroids would be useful for pre-in vivo drug screening during development. Similarly, others have reported that drug diffusion through cells is reduced in 3D spheroid $[59,60]$.

\section{Concluding remarks}

Cells naturally grow in a 3D environment. The spatial arrangement of cells within this environment, including the ECM, affects how they interact with each other and their microenvironment. In turn, these signals affect proliferation, differentiation, morphology and a range of cellular functions $[9,10]$. Therefore, when drug candidates are being tested using cell-based assays, the culture methods used should mimic the most natural in vivo representative form possible. The most natural, tissue-mimicking method of cell growth for drug discovery applications is, arguably, 3D. The drug development process requires further refinement as regards to screening anti-cancer therapeutics [61] to make the process more cost-efficient and to sieve out poorly performing compounds at the earliest stage possible. It appears that the best way to improve on this situation is to improve in vitro screening of candidates by routinely incorporating the analysis of $3 \mathrm{D}$ cell spheroids into the process.

$3 \mathrm{D}$ cell culture is an evolving field and requires further research for its optimisation. While a number of methods for multi-cellular 3D spheroid generation exist, it appears that different cell lines react differently when cultured using the same method. For example MCF7 cells form tight 3D spheroids when grown over p-HEMAcoated plates, while SKBR3 cells only form loose aggregates [20]. It is, therefore, evident that some clarity is needed regarding what method is best for generation of 3D spheroids from individual cell lines. Additionally, the best established 3D culture methods currently available produce avascular tumour models that partly mimic the architecture of in vivo tissues, but leave out the vascularisation aspect of tumour development; which is a hugely significant part of true tumorigenesis. While some groups have recently reported methods for producing $3 \mathrm{D}$ cultures that can incorporate angiogenesis, these methods are limited and are yet 
to be widely established as a technique suitable for in vitro highthroughput drug analysis $[62,63]$. Thus, the emergence of methods that incorporate the angiogenesis process will, undoubtedly, provide better means of mimicking real tumours and such spheroids would, arguably, provide more meaningful data if used in drug discovery/development, drug combination assessment and drugscheduling. Ideally, such 3D cultures would include co-culturing with other relevant cells that are present in human tumours and would be further developed to represent in vivo situations in as best a manner as is possible.

To improve and build on the current drug development process, new ideas and methods must be taken on board to achieve success. Both established and emerging methods must be critically evaluated and compared; considering likeness, first and foremost, to real tissue and tumour architecture; as well as ease of applications in high-throughput scenarios; and, of course, costs. While the generation of 3D culture can be more labour-intensive that 2D culture, the routine incorporation of these multi-cellular spheroids into in vitro drug efficacy and toxicity testing during development will very probably generate more accurate results than the use of monolayer cultures alone, and should better indicate in a timely manner which candidate compounds will/will not have the desired effects on target cells. It is feasible to propose that the routine inclusion of 3D cultures will effectively bridge the gap between in vitro 2D assessment and animal models of disease, fasttracking drug screening and, hopefully, yielding more effective and less toxic drugs as future therapies.

\section{Acknowledgements}

The authors wish to acknowledge funding support in the form of the Marie Keating Foundation PhD Scholarship at Trinity College Dublin. We also thank Science Foundation Ireland (MTCI-SRC/08/B1410).

\section{References}

1 Arrondeau, J. et al. (2010) Development of anti-cancer drugs. Discov. Med. 10, 355 362

2 Hait, W.N. (2010) Anticancer drug development: the grand challenges. Nat. Rev. Drug Discov. 9, 253-254

3 Hopkins, A.L. (2008) Network pharmacology: the next paradigm in drug discovery. Nat. Chem. Biol. 4, 682-690

4 Kola, I. (2008) The state of innovation in drug development. Clin. Pharmacol. Ther. 83, 227-230

5 DiMasi, J.A. and Grabowski, H.G. (2007) Economics of new oncology drug development. J. Clin. Oncol. 25, 209-216

6 Mazzoleni, G. et al. (2009) Modelling tissues in 3D: the next future of pharmacotoxicology and food research? Genes Nutr. 4, 13-22

7 Kunz-Schughart, L.A. et al. (2004) The use of 3-D cultures for high-throughput screening: the multicellular spheroid model. J. Biomol. Screen. 9, 273-285

8 Bhadriraju, K. and Chen, C.S. (2002) Engineering cellular microenvironments to improve cell-based drug testing. Drug Discov. Today 7, 612-620

9 Bissell, M.J. et al. (2003) Tissue architecture: the ultimate regulator of breast epithelial function. Curr. Opin. Cell Biol. 15, 753-762

10 Weaver, V.M. et al. (1997) Reversion of the malignant phenotype of human breast cells in three-dimensional culture and in vivo by integrin blocking antibodies. J. Cell Biol. 137, 231-245

11 Abbott, A. (2003) Cell culture: biology's new dimension. Nature 424, 870-872

12 Bissell, M.J. and Radisky, D. (2001) Putting tumours in context. Nat. Rev. Cancer 1, 46-54

13 Debnath, J. and Brugge, J.S. (2005) Modelling glandular epithelial cancers in threedimensional cultures. Nat. Rev. Cancer 5, 675-688

14 Yamada, K.M. and Cukierman, E. (2007) Modeling tissue morphogenesis and cancer in 3D. Cell 130, 601-610

15 Harrison, R.G. et al. (1907) Observations of the living developing nerve fiber. Anat. Rec. 1, 116-128

16 Harrison, R.G. (1910) The outgrowth of the nerve fiber as a mode of protoplasmic movement. J. Exp. Zool. 9, 787-846

17 Bissell, M.J. et al. (2002) The organizing principle: microenvironmental influences in the normal and malignant breast. Differentiation 70, 537-546

18 O'Brien, L.E. et al. (2002) Building epithelial architecture: insights from threedimensional culture models. Nat. Rev. Mol. Cell Biol. 3, 531-537

19 Lin, R.Z. and Chang, H.Y. (2008) Recent advances in three-dimensional multicellular spheroid culture for biomedical research. Biotechnol. J. 3, 1172-1184

20 Ivascu, A. and Kubbies, M. (2006) Rapid generation of single-tumor spheroids for high-throughput cell function and toxicity analysis. J. Biomol. Screen. 11, 922-932

21 Friedrich, J. et al. (2009) Spheroid-based drug screen: considerations and practical approach. Nat. Protoc. 4, 309-324

$22 \mathrm{Li}$, Q. et al. (2011) 3D models of epithelial-mesenchymal transition in breast cancer metastasis. J. Biomol. Screen. 16, 141-154

23 Oda, Y. et al. (2010) Induction of pluripotent stem cells from human third molar mesenchymal stromal cells. J. Biol. Chem. 285, 29270-29278

24 Morizane, A. et al. (2011) Small-molecule inhibitors of bone morphogenic protein and activin/nodal signals promote highly efficient neural induction from human pluripotent stem cells. J. Neurosci. Res. 89, 117-126
25 Kelm, J.M. et al. (2003) Method for generation of homogeneous multicellular tumor spheroids applicable to a wide variety of cell types. Biotechnol. Bioeng. 83, $173-180$

26 Kurosawa, H. (2007) Methods for inducing embryoid body formation: in vitro differentiation system of embryonic stem cells. J. Biosci. Bioeng. 103, 389-398

27 Tung, Y-C. et al. (2011) High-throughput 3D spheroid culture and drug testing using a 384 hanging drop array. Analyst 136, 473-478

28 Kim, J.B. (2005) Three-dimensional tissue culture models in cancer biology. Semin. Cancer Biol. 15, 365-377

29 Goodwin, T.J. et al. (1993) Reduced shear stress: a major component in the ability of mammalian tissues to form three-dimensional assemblies in simulated microgravity. J. Cell. Biochem. 51, 301-311

30 Rodday, B. et al. (2011) Semiautomatic growth analysis of multicellular tumor spheroids. J. Biomol. Screen. 16, 1119-1124

31 Hirschhaeuser, F. et al. (2009) Test system for trifunctional antibodies in 3D MCTS culture. J. Biomol. Screen. 14, 980-990

32 Barrila, J. et al. (2010) Organotypic 3D cell culture models: using the rotating wall vessel to study host-pathogen interactions. Nat. Rev. Microbiol. 8, 791-801

33 Alcaraz, J. et al. (2004) Biomechanical approaches for studying integration of tissue structure and function in mammary epithelia. J. Mammary Gland Biol. Neoplasia 9, 361-374

34 Kleinman, H.K. and Martin, G.R. (2005) Matrigel: basement membrane matrix with biological activity. Semin. Cancer Biol. 15, 378-386

35 Lee, G.Y. et al. (2007) Three-dimensional culture models of normal and malignant breast epithelial cells. Nat. Methods 4, 359-365

36 Sodunke, T.R. et al. (2007) Micropatterns of Matrigel for three-dimensional epithelial cultures. Biomaterials 28, 4006-4016

37 Sourla, A. et al. (1996) Three-dimensional type I collagen gel system containing MG63 osteoblasts-like cells as a model for studying local bone reaction caused by metastatic cancer cells. Anticancer Res. 16, 2773-2780

38 Glicklis, R. et al. (2000) Hepatocyte behavior within three-dimensional porous alginate scaffolds. Biotechnol. Bioeng. 67, 344-353

39 Tan, W. et al. (2001) Evaluation of nanostructured composite collagen-chitosan matrices for tissue engineering. Tissue Eng. 7, 203-210

40 Justice, B.A. et al. (2009) 3D cell culture opens new dimensions in cell-based assays. Drug Discov. Today 14, 102-107

41 Schmidt, J.J. et al. (2008) Hydrogels used for cell-based drug delivery. J. Biomed. Mater. Res. Part A 87A, 1113-1122

$42 \mathrm{Ji}, \mathrm{C}$. et al. (2011) Enhancing cell penetration and proliferation in chitosan hydrogels for tissue engineering applications. Biomaterials 32, 9719-9729

43 Lee, P.J. et al. (2007) Microfluidic system for automated cell-based assays. J. Assoc. Lab. Autom. 12, 363-367

$44 \mathrm{Wu}$, M.H. et al. (2010) Microfluidic cell culture systems for drug research. Lab Chip 10, 939-956

45 Toh, Y.C. et al. (2007) A novel 3D mammalian cell perfusion-culture system in microfluidic channels. Lab Chip 7, 302-309

46 Zervantonakis, I. et al. (2010) Concentration gradients in microfluidic 3D matrix cell culture systems. Int. J. Micro-Nano Scale Transp. 1, 27-36 
47 Gao, Y. et al. (2011) A versatile valve-enabled microfluidic cell co-culture platform and demonstration of its applications to neurobiology and cancer biology. Biomed. Microdevices 13, 539-548

48 Huh, D. et al. (2011) From 3D cell culture to organs-on-chips. Trends Cell Biol. 21, 745-754

49 Chen, S.Y. et al. (2011) Microfluidic array for three-dimensional perfusion culture of human mammary epithelial cells. Biomed. Microdevices 13, 753-758

50 Slamon, D.J. et al. (1989) Studies of the HER-2/neu proto-oncogene in human breast and ovarian cancer. Science 244, 707-712

51 Pickl, M. and Ries, C.H. (2008) Comparison of 3D and 2D tumor models reveals enhanced HER2 activation in 3D associated with an increased response to trastuzumab. Oncogene 28, 461-468

52 Wilson, W.R. and Hay, M.P. (2011) Targeting hypoxia in cancer therapy. Nat. Rev Cancer 11, 393-410

53 Vaupel, P. and Mayer, A. (2005) Hypoxia and anemia: effects on tumor biology and treatment resistance. Transfus. Clin. Biol. 12, 5-10

54 Weaver, V.M. et al. (2002) [beta] 4 integrin-dependent formation of polarized threedimensional architecture confers resistance to apoptosis in normal and malignant mammary epithelium. Cancer Cell 2, 205-216
55 Li, Q. et al. (2010) Three-dimensional overlay culture models of human breast cancer reveal a critical sensitivity to mitogen-activated protein kinase kinase inhibitors. J. Pharmacol. Exp. Ther. 332, 821-828

56 David, L. et al. (2008) Hyaluronan hydrogel: an appropriate three-dimensional model for evaluation of anticancer drug sensitivity. Acta Biomater. 4, 256-263

57 Ivascu, A. and Kubbies, M. (2007) Diversity of cell-mediated adhesions in breast cancer spheroids. Int. J. Oncol. 31, 1403-1413

58 Thurber, G.M. and Wittrup, K.D. (2008) Quantitative spatiotemporal analysis of antibody fragment diffusion and endocytic consumption in tumor spheroids. Cancer Res. 68, 3334-3341

59 Smalley, K. et al. (2006) Life ins't flat: taking cancer biology to the next dimension. In Vitro Cell. Dev. Biol. Anim. 42, 242-247

60 Lee, J. et al. (2009) Engineering liver tissue spheroids with inverted colloidal crystal scaffolds. Biomaterials 30, 4687-4694

61 Kola, I. and Landis, J. (2004) Can the pharmaceutical industry reduce attrition rates? Nat. Rev. Drug Discov. 3, 711-715

62 Correa de Sampaio, P. et al. (2012) A heterogeneous in vitro three dimensional model of tumour-stroma interactions regulating sprouting angiogenesis. PLoS One 7, E30753

63 Hutmacher, D.W. et al. (2009) Translating tissue engineering technology platforms into cancer research. J. Cell. Mol. Med. 13, 1417-1427 